\title{
Madurez neuropsicológica en preescolares: propiedades psicométricas del test CUMANIN
}

\section{Neuropsychological maturity in preschool children: psychometric properties of CUMANIN test}

\author{
Alfonso Urzúa, Miguel Ramos, Carolina Alday, Alejandro Alquinta \\ Escuela de Psicología, Universidad Católica del Norte, Chile
}

(Rec: 13 mayo 2009 / Acep: 10 octubre 2009)

\begin{abstract}
Resumen
En una muestra de 243 infantes (119 niños y 124 niñas), de edades comprendidas entre 36 y 72 meses, pertenecientes a instituciones públicas y privadas, se evaluaron las propiedades psicométricas post adaptación y normalización del Cuestionario de Madurez Neuropsicológica Infantil CUMANIN. Los resultados encontrados permiten indicar que el instrumento posee una buena consistencia interna y es capaz de discriminar entre rangos de edad, obteniéndose rendimientos más altos a medida que la edad avanza. No se encontraron diferencias en puntajes por sexo. Factores tales como nivel socioeconómico y tipo de institución educacional a la que asiste el párvulo determinaron diferencias en los resultados obtenidos. Se concluye que el Cuestionario de Madurez Neuropsicológica Infantil CUMANIN es un instrumento válido y confiable para la evaluación del constructo madurez neuropsicológica en población preescolar.

Palabras clave: Madurez Neuropsicológica, Evaluación Neuropsicológica Preescolar, CUMANIN
\end{abstract}

\begin{abstract}
In a sample of 243 children (119 males and 124 females) aged between 36 and 72 months, students from public and private institutions, was evaluated the psychometric properties of Child Neuropsychological Maturity Questionnaire CUMANIN, post their adjustment and normalization. These findings can indicate that the instrument has good internal consistency and is able to discriminate between age groups, resulting in higher yields as age advances. There were no differences in scores by gender. Factors such as socioeconomic status and type of educational institution attended by the infant determined differences in the results. We concluded that the Child Neuropsychological Maturity Questionnaire CUMANIN is a reliable and valid instrument for the assessment of neuropsychological maturity construct in preschool population.
\end{abstract}

Key words: Neuropsychological development, neuropsychological evaluation, CUMANIN 


\section{Introducción}

La madurez neuropsicológica se define como el nivel de organización y desarrollo madurativo que permite el desenvolvimiento de las funciones cognitivas y conductuales de acuerdo a la edad cronológica del sujeto (Portellano, Mateos \& Martínez, 2000). En este proceso de desarrollo, la maduración del sistema nervioso central requiere de una secuencia de procesos más compleja que otras estructuras nerviosas, haciendo a este sistema particularmente vulnerable a influencias del ambiente (Rodier, 1994, 2004, en Dietrich et al., 2005), principalmente durante la edad preescolar y escolar, generándose por medio de la interacción entre el ambiente y el sistema nervioso cambios a nivel funcional y estructural (Huttenlocher \& Dabholkar, 1997; Korkman, 2001), tales como la adquisición de las habilidades cognitivas básicas y el conocimiento de la cultura, además de lograr internalizar los patrones conductuales, motivos y valores de un contexto sociocultural particular (Akhutina, Vygotsky \& Luria, 2002; Korkman, 2001; Luria, 1984; Martínez, 1999; Vygotsky, 1981).

La evaluación de la madurez neuropsicológica se realiza por medio de la administración de instrumentos que miden las distintas áreas de funcionamiento cerebral, específicamente de las funciones cognitivas superiores (Silver et al, 2006), existiendo consenso en la investigación de que las principales funciones cognitivas a evaluar son la atención, el lenguaje, las funciones sensoriomotrices, funciones perceptuales y memoria, además de la asimetría funcional o lateralización (Korkman, 2001).

Los instrumentos utilizados en la actualidad, en su mayoría están diseñados para la evaluación de funciones específicas, cuyo propósito es determinar los patrones de desarrollo cerebral, relacionados a fortalezas y debilidades específicas de las funciones neuropsicológicas, para comprender el origen de las dificultades cognitivas y proveer una intervención y tratamiento (D'Amato, Rothlisberg \& Work, 1998). Mientras más completa sea la evaluación, más fácil será comprender el modo en que las funciones neuropsicológicas se encuentran afectadas por patologías, además, de identificar el nivel y calidad de las funciones preservadas en miras a una futura intervención, la cual deberá realizarse programada en base a los resultados obtenidos en la evaluación (Paterno \& Eusebio, 2002).

Aún cuando existen muchas baterías neuropsicológicas desarrolladas para la evaluación de niños de habla anglosajona, pocas baterías de evaluación de las funciones cognitivas y su correlato con el desarrollo cerebral, han sido desarrolladas para niños hispanohablantes (Rosselli, Ardila, Bateman \& Guzmán, 2001).

Específicamente para la población chilena no existen baterías normalizadas que evalúen el constructo madurez neuropsicológica de forma global en el rango etáreo comprendido entre los 3 y 6 años, sino más bien, baterías que evalúan aspectos parciales de la maduración de las funciones cognitivas, lo cual resulta crítico si se considera que una eventual anomalía silenciosa en el desarrollo de algunas de las funciones cognitivas en dicha edad puede tener efectos transcurridos varios años después, concretamente en problemas de aprendizaje derivados de dislexias, discalculias y disgrafías (Portellano, Mateos \& Martínez, 2000), o como lo señala Thorell \& Wahlsted (2006), aquellos niños con deficiencias en funciones como la memoria de trabajo y la fluidez verbal, a futuro pueden desarrollar un trastorno de hiperactividad con déficit atencional, e incluso, trastornos oposicionista desafiante.

Ante esto, se vuelve necesario contar con instrumentos o baterías que permitan elaborar un perfil de las distintas funciones neuropsicológicas de forma completa, a partir de puntos de corte normalizados en nuestra población. El contar con una herramienta de diagnóstico neuropsicológico en el periodo pre-escolar, permitiría realizar un diagnóstico oportuno que posibilite una intervención específica en aquellas funciones que se encuentren en bajo desarrollo, contando con un mejor pronóstico de recuperación dada la neuroplasticidad del niño, en la recuperación y nivelaciones de las funciones cognitivas específicas que se vean disminuidas por un retraso en el desarrollo neural (Bergado \& Almaguer, 2000; Ginarte, 2007; Maggiolo \& de Barbieri, 1999), además de interrumpir un eventual ciclo intergeneracional de anomalías en el desarrollo de las funciones neuropsicológicas (Serbin \& Karp, 2004).

Uno de los instrumentos que han comenzado a utilizarse con fines investigativos es la batería de madurez neuropsicológica - CUMANIN, desarrollada en España por Portellano, Mateos \& Martínez (2000), la cual evalúa las funciones neuropsicológicas en niños entre 3 a 6 años de forma completa. Las propiedades psicométricas de este instrumento ya han comenzado a ser reportadas a nivel sudamericano, como es el caso de la población peruana (Guerrero, 2006), quien obtuvo baremos normativos para niños en edad pre-escolar, concluyendo que el Cuestionario de Madurez Neuropsicológica Infantil CUMANIN, es un test válido y confiable para la evaluación del constructo unidimensional de desarrollo neuropsicológico. Tanto la versión original de Portellano (2000), como la normalización efectuada por Guerrero en Perú, dadas las características socioculturales, ambientales, educativas y psicológicas de cada país, no son válidas en nuestra población, por lo que el presente estudio tiene por objeto adaptar y normalizar el instrumento de estimación de la madurez neuropsicológica CUMANIN, en población entre los 3 y 6 años, lo que permitirá contar con una herramienta de evaluación neuropsicológica que posibilite elaborar un diagnóstico más certero y completo a fin de implementar los métodos adecuados para la corrección y la prevención de los problemas comunes en el aprendizaje escolar (Quintanar \& Solovieva, 2005). Al realizar este estudio también se pretende indagar sobre si variables 
como la edad, el sexo, el quintil de ingreso o el tipo de establecimiento educacional al que asisten los pre-escolares -jardín infantil público o privado- tienen incidencia en su nivel de desarrollo neuromadurativo, hipotetizándose que: (a) Existirían diferencias entre los puntajes obtenidos en el test CUMANIN por pre-escolares pertenecientes a distinto rango etáreo, esperándose un mejor desempeño a medida que avanzan en edad, (b) No existirían diferencias entre los puntajes obtenidos por pre-escolares hombres y mujeres en menores de 6 años (c) Existirían diferencias entre los puntajes obtenidos en el test CUMANIN por pre-escolares pertenecientes a distinto nivel de ingreso económico, esperándose mejores puntajes en niveles de ingreso superior, (d) Existirían diferencias entre los puntajes obtenidos por pre-escolares pertenecientes a distinto tipo de establecimiento educacional (jardín infantil público o privado), suponiéndose encontrar un mejor desempeño en los establecimientos privados.

\section{Método}

\section{Participantes}

La muestra estuvo compuesta por 243 participantes, de los cuales $119(49 \%)$ fueron hombres y 124 (51\%) mujeres, los cuales fueron seleccionados de manera no aleatoria e intencionada de jardines infantiles pertenecientes a la Junta Nacional de Jardines Infantiles (JUNJI) y también de Jardines Particulares de la ciudad de Antofagasta, todos ellos inscritos en la base de datos de establecimientos de educación preescolar de la ciudad perteneciente a la JUNJI, a fin de considerar en la muestra participantes de toda la ciudad.

A partir de los datos entregados por la JUNJI sobre la cantidad de jardines infantiles por zona geográfica, se evaluaron participantes de 08 jardines de la zona norte, 10 de la zona centro y 03 de la zona sur, considerando para la elección de los jardines el quintil de ingreso promedio de los establecimientos, según datos reportados por los directores de cada establecimiento educacional.

La edad de los evaluados fluctuó entre los 36 y los 72 meses, con una media de 53.82 meses ( $\mathrm{DT}=9.47$ ). Dada la edad de ingreso a la educación básica en Chile (6 años) no fue posible acceder a un número suficiente de participantes pertenecientes al último rango de edad establecido por Portellano para la muestra española, por lo que la muestra obtenida cuyas edades fluctuaban entre 73 y 78 meses fue eliminada de los análisis estadísticos, quedando la muestra final integrada por 243 participantes.

La distribución final de los sujetos según rango de edad puede ser observada en la tabla 1 .

\section{Procedimiento}

El proceso de levantamiento de datos pudo ser realizado una vez que se hubo obtenido el permiso de la Junta Nacional de Jardines Infantiles de Antofagasta (JUNJI) para realizar la investigación.

La prueba fue sometida inicialmente a una aplicación piloto en 20 menores y a una revisión por expertos a fin de ser modificada y adaptada a las características de la población pre-escolar antofagastina. La prueba así como la tablilla de tabulación de las respuestas fueron modificadas y este nuevo formato se aplicó a una muestra de 251 niños, de manera individual en los establecimientos educacionales a los cuales asistían los niños. Se solicitó previamente la autorización a participar en el estudio a los encargados de los establecimientos educacionales en los que se realizó la aplicación del instrumento, mediante una carta de consentimiento escrito.

Los evaluadores fueron seleccionados del grupo de neuropsicología de la Escuela de Psicología de la Universidad Católica del Norte, y capacitados en el uso del test CUMANIN, sus características y su metodología de aplicación, realizándose al final una evaluación piloto

Tabla 1. Distribución de la frecuencia y porcentaje de la muestra según edad en meses, tipo de institución y sexo.

\begin{tabular}{ccccccccccccccc}
\hline \multirow{2}{*}{ Rango meses } & \multicolumn{4}{c}{ JUNJI } & \multicolumn{4}{c}{ M } & \multicolumn{4}{c}{ Particulares } & \multicolumn{4}{c}{ Total } \\
& \multicolumn{2}{c}{ Hombres } & \multicolumn{2}{c}{ Mujeres } & \multicolumn{2}{c}{ Subtotal } & \multicolumn{2}{c}{ Hombres } & \multicolumn{2}{c}{ Mujeres } & \multicolumn{2}{c}{ Subtotal } & \multicolumn{2}{c}{ Total } \\
& $\mathrm{n}$ & $\%$ & $\mathrm{n}$ & $\%$ & $\mathrm{n}$ & $\%$ & $\mathrm{n}$ & $\%$ & $\mathrm{n}$ & $\%$ & $\mathrm{n}$ & $\%$ & $\mathrm{n}$ & $\%$ \\
\hline $36-42$ & 6 & 10,34 & 10 & 17,24 & 16 & 13,79 & 10 & 16,39 & 7 & 10,61 & 17 & 13,39 & 33 & 13,6 \\
$43-48$ & 8 & 13,79 & 4 & 6,90 & 12 & 10,34 & 15 & 24,59 & 29 & 43,94 & 44 & 34,65 & 56 & 23,0 \\
$49-54$ & 9 & 15,52 & 12 & 20,69 & 21 & 18,10 & 12 & 19,67 & 3 & 4,55 & 15 & 11,81 & 36 & 14,8 \\
$55-60$ & 15 & 25,86 & 12 & 20,69 & 27 & 23,28 & 10 & 16,39 & 14 & 21,21 & 24 & 18,90 & 51 & 21,0 \\
$61-66$ & 14 & 24,14 & 14 & 24,14 & 28 & 24,14 & 10 & 16,39 & 7 & 10,61 & 17 & 13,39 & 45 & 18,5 \\
$67-72$ & 6 & 10,34 & 6 & 10,34 & 12 & 10,34 & 4 & 6,56 & 6 & 9,09 & 10 & 7,87 & 22 & 9,1 \\
Total & 58 & 100 & 58 & 100 & 116 & 100 & 61 & 100 & 66 & 100 & 127 & 100 & 243 & 100 \\
\hline
\end{tabular}


en sala de espejo para unificar y verificar los criterios de puntuación.

El tiempo promedio de respuesta fue de 40 minutos aproximadamente.

Una vez terminadas las evaluaciones, estas fueron ingresadas a una base de datos en SPSS 11.5 para su posterior análisis estadístico.

\section{Instrumento}

El Cuestionario de Madurez Neuropsicológica Infantil - CUMANIN está conformado por 83 ítems agrupados en 13 sub-escalas, cada ítem es valorado como acierto (1) o error $(0)$, recogiendo también información respecto a lateralidad de mano, ojo y pie. Las 8 escalas principales son: Psicomotricidad, Lenguaje articulatorio, Lenguaje comprensivo, Lenguaje expresivo, Estructuración espacial, Viso percepción, Memoria y Ritmo.

La Escala de Psicomotricidad (11 ítems), constituida por siete tareas permite un máximo de 12 puntos con tareas como por ejemplo caminar en un pie, tocar la nariz con el dedo o andar en equilibrio. La escala de Lenguaje Articulatorio (15 ítems) se constituye en la repetición de 15 palabras de dificultad articulatoria creciente. La Escala de Lenguaje Expresivo (4 ítems) consiste en la repetición de cuatro frases de dificultad creciente. La Escala de Lenguaje Comprensivo (9 ítems), consiste en la lectura de un texto al niño y el planteamiento de nueve preguntas sobre su contenido. La Escala de Estructuración Espacial (15 ítems) está conformada por 15 tareas que deben ser ejecutadas con respuestas psicomotoras y grafo motoras. La Escala de Viso percepción (15 ítems) consiste en la reproducción de 15 modelos geométricos de complejidad creciente. La Escala de Memoria Icónica consiste en la presentación visual de una lámina con grabados de objetos sencillos, los que deben ser memorizados por el examinado. La Escala de Ritmo (7 ítems), consiste en la reproducción de 7 series rítmicas con aumento gradual de la dificultad, las que son presentadas auditivamente por el examinador.

El instrumento consta además de 5 escalas adicionales, las cuales no forman parte del conjunto destinado a medir desarrollo neuropsicológico. Dos de ellas evalúan las áreas de Atención y Fluidez verbal, y a partir de los 5 años (60 meses), se pueden aplicar dos escalas de lenguaje escrito: Lectura y Escritura.

Cada escala permite registrar puntuaciones cuya interpretación se hace convirtiendo estos puntajes brutos en escalas centiles, los que están diferenciados en 5 grupos de edad en meses. Permitiendo además obtener un perfil de resultados.

La puntuación total (Desarrollo Global), formada por los 83 ítems de las 8 escalas principales, se interpreta en centiles, y de esta se puede obtener un índice en términos de cociente de desarrollo (CD). La prueba permite además obtener puntuaciones y centiles del Desarrollo Verbal y Desarrollo no-verbal del sujeto, los que se obtienen de las 8 escalas principales.

La validación del instrumento español se realizó con 803 niños, el análisis estadístico incluyó análisis de ítems en el enfoque de la teoría clásica de los Test y dentro de la Teoría de Respuesta al Ítem, cálculos de análisis factorial exploratorio y correlaciones tetracóricas, que apoyaron la unidimensionalidad de la escala. Los valores del coeficiente $\alpha$ de Cronbach obtenido por los autores del instrumento fluctuaron entre 0.71 y 0.92 .

El instrumento ha sido utilizado en Lima, Perú, en donde se aplicó a una muestra de 261 niños desde cuarenta y dos meses a setenta y ocho meses de edad de centros de educación inicial particulares y nacionales. Se analizaron los ítems por dificultad y discriminación; confiabilidad con $\alpha$ de Cronbach; validez determinada por: criterio de jueces, constructo y análisis factorial; baremos. Además se encontró que los coeficientes $\alpha$ para las escalas de Psicomotricidad, Lenguajes Articulatorio, Expresivo y Comprensivo, Estructuración Espacial, Viso percepción, Memoria y Ritmo, fluctúan entre 0.51 y -0.87 . La media por escala incrementó progresivamente con la edad, con diferencias significativas entre grupos. Finalmente en el análisis factorial las escalas saturaron en un factor apoyando la unidimensionalidad del constructo madurez neuropsicológica.

\section{Análisis Estadísticos}

Para evaluar la consistencia interna del CUMANIN se utilizó el $\alpha$ de Cronbach. A fin de determinar si existían diferencias en las puntuaciones de la muestra relacionadas al sexo se realizó un análisis de prueba $\mathrm{T}$ para muestras independientes. Para determinar si el factor edad estaba correlacionado con las puntuaciones obtenidas por la muestra en cada una de las dimensiones se realizó un análisis de coeficiente de correlación bivariada (Pearson).

Debido a que las escalas de: Atención, Fluidez Verbal, Lectura y Escritura no forman parte del conjunto destinado a medir el desarrollo neuropsicológico, no fueron incluidas en los análisis estadísticos de fiabilidad ( $\alpha$ de Cronbach) al igual que la investigación original realizada en España y en la adaptación y validación del instrumento realizada en Lima, Perú.

\section{Resultados}

\section{Consistencia Interna}

Tal como se puede observar en la tabla 2, todas las dimensiones evaluadas presentan una consistencia interna entre moderada y buena, siendo los $\alpha$ reportados superiores a .66. 
Tabla 2: $\alpha$ reportado en las muestras de Antofagasta, Lima y Madrid

\begin{tabular}{lccc}
\hline & $\alpha$ Antofagasta & $\alpha$ Lima & $\alpha$ Madrid \\
\hline Psicomotricidad & 0.66 & 0.64 & 0.71 \\
Lenguaje Articulatorio & 0.87 & 0.87 & 0.92 \\
Lenguaje Expresivo & 0.66 & 0.63 & 0.73 \\
Lenguaje Comprensivo & 0.75 & 0.67 & 0.72 \\
Estructuración Espacial & 0.69 & 0.80 & 0.81 \\
Viso percepción & 0.89 & 0.86 & 0.91 \\
Memoria Icónica & 0.69 & 0.51 & 0.57 \\
Ritmo & 0.63 & 0.66 & 0.72 \\
Escala Total & 0.82 & - & - \\
\hline
\end{tabular}

Tabla 3: Correlaciones entre sub-escalas

\begin{tabular}{|c|c|c|c|c|c|c|c|c|c|}
\hline & Psico & Leng. Art. & Leng. Exp. & Leng. Comp. & Estruct. Esp. & Visoper. & Mem. Ico. & Ritmo & Fluid. Verb. \\
\hline Lenguaje Articulatorio & $0,49(*)$ & & & & & & & & \\
\hline Lenguaje expresivo & $0,48(*)$ & $0,49(*)$ & & & & & & & \\
\hline Lenguaje comprensivo & $0,43(*)$ & $0,39(*)$ & $0,48(*)$ & & & & & & \\
\hline Estructuración Espacial & $0,41(*)$ & $0,33(*)$ & $0,35\left(^{*}\right)$ & $0,36(*)$ & & & & & \\
\hline Visopercepción & $0,52(*)$ & $0,53(*)$ & $0,46(*)$ & $0,40(*)$ & $0,44(*)$ & & & & \\
\hline Memoria icónica & $0,47(*)$ & $0,28(*)$ & $0,42(*)$ & $0,35(*)$ & $0,40(*)$ & $0,38(*)$ & & & \\
\hline Ritmo & $0,36(*)$ & $0,45(*)$ & $0,38(*)$ & $0,25\left(^{*}\right)$ & $0,25\left(^{*}\right)$ & $0,56(*)$ & $0,24\left(^{*}\right)$ & & \\
\hline Fluidez verbal & $0,37(*)$ & $0,27(*)$ & $0,40(*)$ & $0,40(*)$ & $0,29(*)$ & $0,33(*)$ & $0,29(*)$ & $0,24(*)$ & \\
\hline Atención & $0,36(*)$ & $0,37(*)$ & $0,36(*)$ & $0,38(*)$ & $0,42(*)$ & $0,46(*)$ & $0,38(*)$ & $0,33(*)$ & $0,251(*)$ \\
\hline
\end{tabular}

\section{Correlaciones entre las escalas}

El análisis de correlación entre las dimensiones de la escala indica que todas están moderadamente relacionadas, ya que todas las correlaciones fueron significativas $(\mathrm{p}<.01)$, tal como se muestra en la tabla 3.

A fin de demostrar la unidimensionalidad de la escala se realizó un análisis factorial de segundo orden, encontrándose que este único factor explicaría el 45,16 \% de la varianza total, teniendo todas las sub-escalas saturaciones moderadas o altas en éste. Estos hallazgos como se observa en la tabla 4, guardan congruencia con los resultados obtenidos en las investigaciones realizadas tanto en Madrid, como en Lima.

Como se observa, el conjunto de sub-escalas incluidas las adicionales de Atención y Fluidez verbal, también pueden corresponden al factor único medido por el test CUMANIN.

\section{Madurez Neuropsicológica}

La tabla 5 nos muestra las medias obtenidas en las distintas dimensiones según sexo y tipo de establecimiento educacional.

Al comparar las medias de las diversas áreas obtenidas por hombres y mujeres, no se encuentran diferencias estadísticamente significativas entre éstas. Al comparar los datos obtenidos entre jardines públicos y jardines privados, se observa que existen diferencias estadísticamente significativas en las dimensiones Estructuración Espacial $(\mathrm{t}(241)=-2,340 ; \mathrm{p}>.05)$, en donde la media de los Jardines privados es significativamente más alta que en los establecimientos públicos. También se observan diferencias estadísticamente significativas en las dimensiones Ritmo $(\mathrm{t}(241)=2,324 ; \mathrm{p}>.05)$ y Fluidez Verbal $(\mathrm{t}(241)=2,099$; $\mathrm{p}>.05$ ) en donde las medias de los jardines públicos son más altas que en los jardines privados.

Al analizar la relación existente con la edad, se observa que ésta, tal como se esperaba, correlaciona significativamente con cada una de las dimensiones evaluadas, tal como se observa en la tabla 6. 
Tabla 4: Saturación de las subescalas con el factor principal

\begin{tabular}{lccc}
\hline & Saturación (Antofagasta) & Saturación (Madrid) & Saturación (Lima) 2006 \\
\hline Psicomotricidad & 2007 & 2002 & 0,70 \\
Lenguaje Articulatorio & 0,73 & 0,72 & 0,67 \\
Lenguaje Expresivo & 0,70 & 0,70 & 0,71 \\
Lenguaje Comprensivo & 0,73 & 0,83 & 0,69 \\
Estructuración Espacial & 0,67 & 0,73 & 0,69 \\
Visopercepción & 0,63 & 0,68 & 0,73 \\
Memoria Icónica & 0,77 & 0,79 & 0,47 \\
Ritmo & 0,61 & 0,67 & 0,64 \\
Fluidez Verbal & 0,61 & 0,51 & 0,52 \\
Atención & 0,56 & 0,59 & \\
\hline
\end{tabular}

Tabla 5: Medias reportadas según sexo y según tipo de establecimiento educacional

\begin{tabular}{|c|c|c|c|c|}
\hline & Hombres & Mujeres & JUNJI & Privados \\
\hline Psicomotricidad & 6.93 & 7.35 & 7.34 & 6.96 \\
\hline Lenguaje Articulatorio & 8.51 & 9.41 & 8.58 & 9.32 \\
\hline Lenguaje Expresivo & 2.18 & 2.30 & 2.23 & 2.25 \\
\hline Lenguaje Comprensivo & 3.72 & 3.84 & 3.77 & 3.79 \\
\hline Estructuración Espacial & 8.01 & 7.45 & 7.29 & 8.13 \\
\hline Viso percepción & 6.22 & 5.62 & 5.81 & 6.02 \\
\hline Memoria Icónica & 5.78 & 6.16 & 6.26 & 5.71 \\
\hline Ritmo & 1.98 & 1.91 & 2.18 & 1.74 \\
\hline Fluidez Verbal & 54.05 & 43.57 & 58.55 & 39.71 \\
\hline Atención & 6.66 & 7.19 & 7.15 & 6.73 \\
\hline Lectura & 0.16 & 0.10 & 0.00 & 0.25 \\
\hline Escritura & 0.11 & 0.06 & 0.00 & 0.17 \\
\hline
\end{tabular}

Tabla 6: Correlación edad y puntaje en dimensiones $(n=243)$

\begin{tabular}{|c|c|c|c|c|c|}
\hline Dimensión & $\mathrm{r}$ & Sig. & Dimensión & $\mathrm{r}$ & $\mathrm{p}$ \\
\hline Psicomotricidad & 0.49 & 0.00 & Memoria Icónica & 0.37 & 0.00 \\
\hline Lenguaje Articulatorio & 0.48 & 0.00 & Ritmo & 0.43 & 0.00 \\
\hline Lenguaje Expresivo & 0.49 & 0.00 & Fluidez Verbal & 0.38 & 0.00 \\
\hline Lenguaje Comprensivo & 0.44 & 0.00 & Atención & 0.47 & 0.00 \\
\hline Estructuración Espacial & 0.37 & 0.00 & Lectura & 0.17 & 0.007 \\
\hline Viso percepción & 0.73 & 0.00 & Escritura & 0.16 & 0.011 \\
\hline
\end{tabular}

A fin de evaluar si existían diferencias entre las medias encontradas en cada rango de edad para cada uno de los dominios, se realiza un análisis de varianza (ANOVA), encontrándose diferencias estadísticamente significativas entre las medias de los rangos de edad en todos los dominios (Tabla 7).

Al evaluar la homogeneidad de las varianzas a través de la prueba de Levene, se encuentra que no existe homogeneidad de éstas en los dominios lenguaje expresivo $(\mathrm{p}<0,001)$, lenguaje comprensivo $(\mathrm{p}=0,003)$, viso percepción $(\mathrm{p}=0,001)$, memoria icónica $(\mathrm{p}=0,032)$, fluidez verbal $(\mathrm{p}<0,001)$, lectura $(\mathrm{p}<0,001)$ y escritura $(\mathrm{p}<0,001)$, por lo que en estas se realizaron contrastes post hoc a través de la prueba de Games-Howell, a diferencia de los dominios donde sí existía homogeneidad en donde se analizaron las diferencias con la prueba HSD de Tukey (Tabla 7). 
Tabla 7. ANOVA medias, dimensiones y rango de meses

\begin{tabular}{|c|c|c|c|c|c|c|c|c|c|c|}
\hline & $\begin{array}{c}\text { Rango } \\
\text { de meses }\end{array}$ & $\mathrm{N}$ & Media & $\begin{array}{c}\text { Desviación } \\
\text { típica }\end{array}$ & & $\begin{array}{c}\text { Suma de } \\
\text { cuadrados }\end{array}$ & $\mathrm{gl}$ & $\begin{array}{c}\text { Media } \\
\text { cuadrática }\end{array}$ & $\mathrm{F}$ & $\mathrm{p}$ \\
\hline \multirow{8}{*}{ Psicomotricidad } & $36-42$ & 33 & 5,606 & 1,903 & Inter-grupos & 284,487 & 5 & 56,897 & 17,739 &, 000 \\
\hline & $43-48$ & 56 & 6,518 & 2,166 & Intra-grupos & 760,179 & 237 & 3,208 & & \\
\hline & $49-54$ & 36 & 6,444 & 1,647 & Total & 1044,667 & 242 & & & \\
\hline & $55-60$ & 51 & 7,275 & 1,72138 & & & & & & \\
\hline & $61-66$ & 45 & 8,6667 & 1,52256 & & & & & & \\
\hline & $67-72$ & 22 & 8,8182 & 1,40192 & & & & & & \\
\hline & $36-42$ & 33 & 4,0000 & 3,34477 & Inter-grupos & 1268,150 & 5 & 253,630 & 19,437 &, 000 \\
\hline & $43-48$ & 56 & 8,3571 & 3,56971 & Intra-grupos & 3092,648 & 237 & 13,049 & & \\
\hline \multirow[t]{5}{*}{ Lenguaje Articulatorio } & $49-54$ & 36 & 8,5556 & 3,58126 & Total & 4360,798 & 242 & & & \\
\hline & $55-60$ & 51 & 10,1176 & 3,84524 & & & & & & \\
\hline & $61-66$ & 45 & 11,2889 & 3,42864 & & & & & & \\
\hline & $67-72$ & 22 & 11,2727 & 3,95428 & & & & & & \\
\hline & $36-42$ & 33 & 1,2727 & 1,30558 & Inter-grupos & 106,888 & 5 & 21,378 & 16,760 &, 000 \\
\hline \multirow[t]{6}{*}{ Lenguaje Expresivo } & $43-48$ & 56 & 1,6250 & 1,38252 & Intra-grupos & 302,297 & 237 & 1,276 & & \\
\hline & $49-54$ & 36 & 2,3889 &, 93435 & Total & 409,185 & 242 & & & \\
\hline & $55-60$ & 51 & 2,3137 & 1,17457 & & & & & & \\
\hline & $61-66$ & 45 & 3,0000 &, 87905 & & & & & & \\
\hline & $67-72$ & 22 & 3,3636 & ,65795 & & & & & & \\
\hline & $36-42$ & 33 & 2,2727 & 1,77258 & Inter-grupos & 313,394 & 5 & 62,679 & 13,389 &, 000 \\
\hline \multirow[t]{6}{*}{ Lenguaje Comprensivo } & $43-48$ & 56 & 3,0000 & 2,51541 & Intra-grupos & 1109,478 & 237 & 4,681 & & \\
\hline & $49-54$ & 36 & 2,8611 & 1,95890 & Total & 1422,872 & 242 & & & \\
\hline & $55-60$ & 51 & 4,5490 & 2,47640 & & & & & & \\
\hline & $61-66$ & 45 & 4,6667 & 2,00000 & & & & & & \\
\hline & $67-72$ & 22 & 6,0000 & 1,44749 & & & & & & \\
\hline & $36-42$ & 33 & 6,0606 & 2,35769 & Inter-grupos & 301,275 & 5 & 60,255 & 8,770 &, 000 \\
\hline \multirow[t]{5}{*}{ Estructuración Espacial } & $43-48$ & 56 & 7,1607 & 2,44783 & Intra-grupos & 1628,338 & 237 & 6,871 & & \\
\hline & $49-54$ & 36 & 6,8056 & 2,40023 & Total & 1929,613 & 242 & & & \\
\hline & $55-60$ & 51 & 8,1176 & 2,80462 & & & & & & \\
\hline & $61-66$ & 45 & 9,2000 & 2,69343 & & & & & & \\
\hline & $67-72$ & 22 & 9,3182 & 3,13788 & & & & & & \\
\hline \multirow{6}{*}{ Viso percepción } & $36-42$ & 33 & 1,8485 & 2,30653 & Inter-grupos & 2224,905 & 5 & 444,981 & 62,602 &, 000 \\
\hline & $43-48$ & 56 & 3,7321 & 2,37786 & Intra-grupos & 1684,610 & 237 & 7,108 & & \\
\hline & $49-54$ & 36 & 3,8333 & 1,96396 & Total & 3909,514 & 242 & & & \\
\hline & $55-60$ & 51 & 7,0784 & 3,09091 & & & & & & \\
\hline & $61-66$ & 45 & 9,2889 & 3,32089 & & & & & & \\
\hline & $67-72$ & 22 & 11,4545 & 2,24090 & & & & & & \\
\hline \multirow{6}{*}{ Memoria icónica } & $36-42$ & 33 & 4,8485 & 2,33347 & Inter-grupos & 221,829 & 5 & 44,366 & 8,843 &, 000 \\
\hline & $43-48$ & 56 & 4,8750 & 2,55174 & Intra-grupos & 1189,068 & 237 & 5,017 & & \\
\hline & $49-54$ & 36 & 6,2778 & 2,34960 & Total & 1410,897 & 242 & & & \\
\hline & $55-60$ & 51 & 6,0588 & 2,38673 & & & & & & \\
\hline & $61-66$ & 45 & 7,1333 & 1,80404 & & & & & & \\
\hline & $67-72$ & 22 & 7,4545 & 1,29935 & & & & & & \\
\hline
\end{tabular}


Continuación Tabla 7

\begin{tabular}{|c|c|c|c|c|c|c|c|c|c|c|}
\hline & $\begin{array}{c}\text { Rango } \\
\text { de meses }\end{array}$ & $\mathrm{N}$ & Media & $\begin{array}{c}\text { Desviación } \\
\text { típica }\end{array}$ & & $\begin{array}{c}\text { Suma de } \\
\text { cuadrados }\end{array}$ & $\mathrm{gl}$ & $\begin{array}{c}\text { Media } \\
\text { cuadrática }\end{array}$ & $\mathrm{F}$ & $\mathrm{p}$ \\
\hline \multirow{6}{*}{ Ritmo } & $36-42$ & 33 & ,9394 & 1,05887 & Inter-grupos & 128,020 & 5 & 25,604 & 14,823 & ,000 \\
\hline & $43-48$ & 56 & 1,7857 & 1,37132 & Intra-grupos & 409,387 & 237 & 1,727 & & \\
\hline & $49-54$ & 36 & 1,2778 & 1,20975 & Total & 537,407 & 242 & & & \\
\hline & $55-60$ & 51 & 1,8824 & 1,55752 & & & & & & \\
\hline & $61-66$ & 45 & 2,8667 & 1,19848 & & & & & & \\
\hline & $67-72$ & 22 & 3,2727 & 1,27920 & & & & & & \\
\hline \multirow[t]{6}{*}{ Fluidez Verbal } & $36-42$ & 33 & 31,6364 & 55,58261 & Inter-grupos & 295402,883 & 5 & 59080,577 & 15,512 &, 000 \\
\hline & $43-48$ & 56 & 21,1250 & 27,67346 & Intra-grupos & 902667,372 & 237 & 3808,723 & & \\
\hline & $49-54$ & 36 & 25,8611 & 39,97367 & Total & 1198070,255 & 242 & & & \\
\hline & $55-60$ & 51 & 60,1961 & 81,57917 & & & & & & \\
\hline & $61-66$ & 45 & 52,2444 & 56,37502 & & & & & & \\
\hline & $67-72$ & 22 & 148,0455 & 105,37076 & & & & & & \\
\hline \multirow{6}{*}{ Atención } & $36-42$ & 33 & 3,6667 & 3,36031 & Inter-grupos & 1432,991 & 5 & 286,598 & 16,035 &, 000 \\
\hline & $43-48$ & 56 & 5,2143 & 5,07656 & Intra-grupos & 4235,955 & 237 & 17,873 & & \\
\hline & $49-54$ & 36 & 4,7778 & 4,25012 & Total & 5668,947 & 242 & & & \\
\hline & $55-60$ & 51 & 8,4118 & 4,38715 & & & & & & \\
\hline & $61-66$ & 45 & 10,0667 & 3,66432 & & & & & & \\
\hline & $67-72$ & 22 & 9,9091 & 3,59774 & & & & & & \\
\hline \multirow{6}{*}{ Lectura } & $36-42$ & 33 &, 0000 &, 00000 & Inter-grupos & 21,065 & 5 & 4,213 & 3,248 & ,007 \\
\hline & $43-48$ & 56 &, 0000 &, 00000 & Intra-grupos & 307,454 & 237 & 1,297 & & \\
\hline & $49-54$ & 36 & ,0000 & ,00000 & Total & 328,519 & 242 & & & \\
\hline & $55-60$ & 51 & ,0392 & ,28006 & & & & & & \\
\hline & $61-66$ & 45 & ,1778 & 1,19257 & & & & & & \\
\hline & $67-72$ & 22 & 1,0455 & 3,38733 & & & & & & \\
\hline \multirow{6}{*}{ Escritura } & $36-42$ & 33 &, 0000 & ,00000 & Inter-grupos & 16,279 & 5 & 3,256 & 3,983 & ,002 \\
\hline & $43-48$ & 56 &, 0000 &, 00000 & Intra-grupos & 193,729 & 237 & 817 & & \\
\hline & $49-54$ & 36 &, 0000 &, 00000 & Total & 210,008 & 242 & & & \\
\hline & $55-60$ & 51 &, 0000 &, 00000 & & & & & & \\
\hline & $61-66$ & 45 &, 0444 & ,29814 & & & & & & \\
\hline & $67-72$ & 22 & ,9091 & 3,00649 & & & & & & \\
\hline
\end{tabular}

Tal como se hipotetizaba, la escala es capaz de discernir entre grupos de edad, ya que tal como se puede observar en la figura 1, las medias en las distintas dimensiones se incrementan a medida que la edad también lo hace.

En la Tabla 8 se observa que las medias obtenidas en la evaluación son significativamente distintas entre Quintiles de Ingreso, para las dimensiones Psicomotricidad, Estructuración Espacial, Viso percepción, Ritmo y Fluidez Verbal, siendo la media en estas superior en el quintil con mayor ingreso.

Esto puede corroborarse al aplicar la prueba post hoc de Games - Howell para la dimensiones psicomotricidad y fluidez verbal, y la HSD de Tukey para estructuración espacial, viso percepción y ritmo, habiéndose evaluado previamente la homogeneidad de la varianza a través de la prueba de Levene (psicomotricidad p $=0,012$; estructuración espacial $\mathrm{p}=0,532$; Viso percepción $\mathrm{p}=0,701 ;$ Ritmo $\mathrm{p}=0,249$ y Fluidez Verbal $\mathrm{p}=0,002$ ).

En la figura 2 puede apreciarse cómo las medias pertenecientes al quintil con mejores ingresos son significativamente superiores.

\section{Discusión}

Los resultados encontrados permiten asegurar una adecuada consistencia interna del instrumento, hecho también reportado en las investigaciones realizadas en la muestra española y la validación en población peruana, siendo el $\alpha$ en todos los estudios superior a .66. Por otra parte, los análisis de correlación entre las sub-escalas permitieron demostrar 


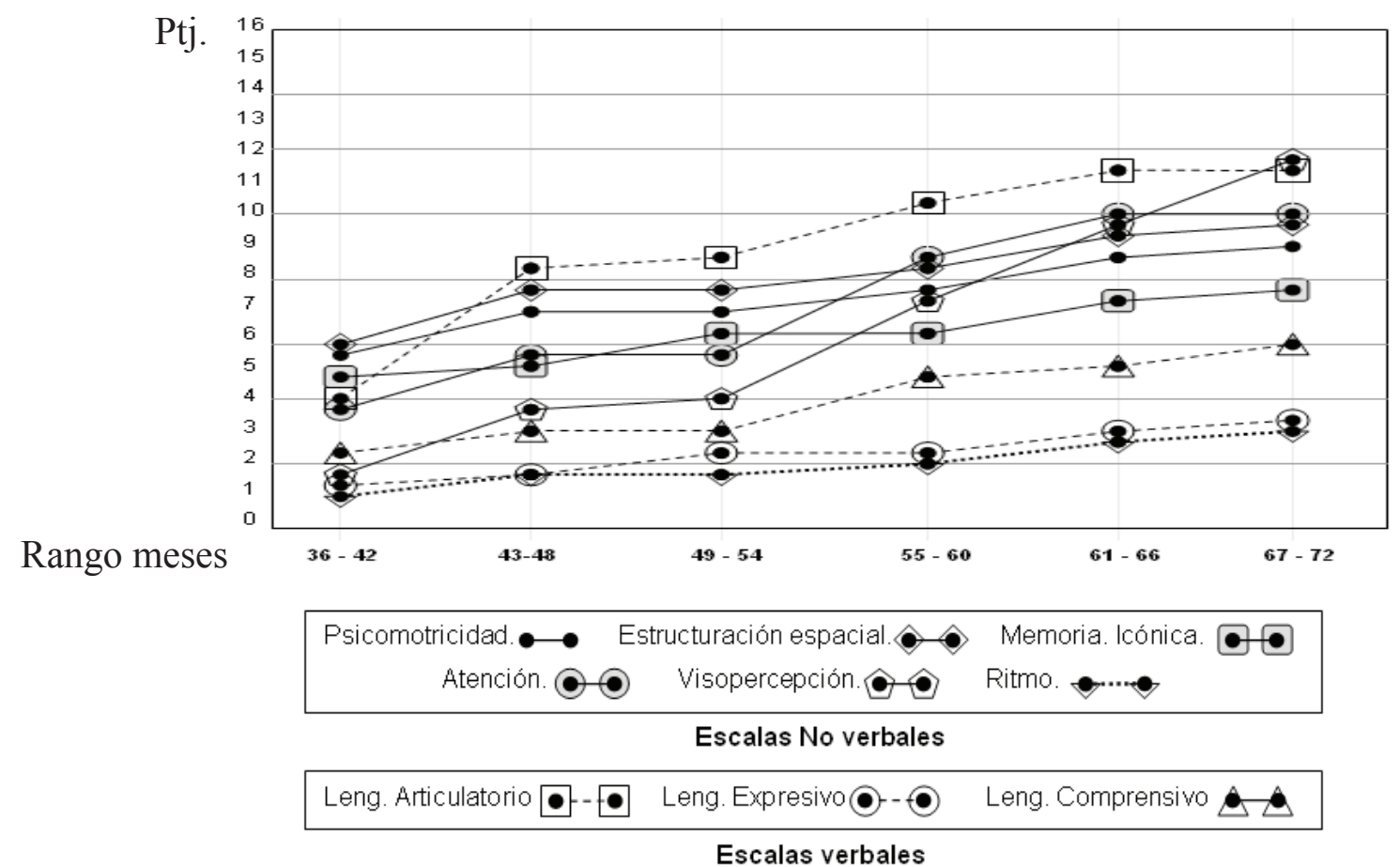

Figura 1: Medias según dimensión y rango etáreo

la unidimensionalidad de la escala total obteniéndose significancia en la correlación al nivel 0.01 en todas estas.

Finalmente la saturación del factor, demostró que tanto las sub-escalas principales como las adicionales corresponden al factor único medido por el instrumento CUMANIN.

Aun cuando no se tienen referencias de la población peruana acerca del impacto o el rol que pudiese tener el sexo en la mayor o menor madurez de ciertas funciones superiores, los hallazgos encontrados en esta investigación, al igual que los reportados por Portellano (2000) indicarían que la condición biológica de ser hombre o ser mujer no sería una variable relacionada con la madurez neuropsicológica. Con relación a este aspecto, Kolb \& Whishaw (2006) mostraron que en pruebas neuropsicológicas de fluidez verbal y percepción visual existen diferencias significativas entre ambos sexos, solo después de los 6 años de edad en adelante. En base a estos antecedentes podría pensarse que podrían existir diferencias en el rendimiento neuropsicológico entre niños y niñas posteriores a la edad pre-escolar y no aquellos rangos etáreos evaluados por el test CUMANIN.

Donde sí se encuentran diferencias estadísticamente significativas es entre las medias reportadas por los jardines infantiles públicos con las medias encontradas en los jardines de carácter privado, siendo en algunas dimensiones superiores en los públicos y en otras en los privados.

La media de la dimensión estructuración espacial es significativamente más alta en los establecimientos privados que en los establecimientos públicos, a diferencia de la media de las dimensiones ritmo y fluidez verbal en donde las medias de los jardines públicos son más altas que en los jardines privados.

En la revisión bibliográfica realizada no se ha encontrado estudios que evalúen el desarrollo neuropsicológico comparando distintos niveles de ingreso ni tipo de establecimiento educacional, sin embargo Solovieva, Quintanar \& Lázaro (2002) realizaron en México un estudio comparativo entre niños pertenecientes a escuelas rurales y niños pertenecientes a escuelas urbanas privadas, consiguiendo como resultado que los niños de escuela rural obtuvieron peores resultados que los pertenecientes a escuelas privadas en todas las dimensiones y no en algunas en particular.

Una posible hipótesis que permitiese explicar tales diferencias puede ser el tipo de metodología utilizada en los jardines públicos, en donde las actividades realizadas generen un mayor desarrollo de las funciones vinculadas al ritmo, o bien por procesos de socialización del lenguaje distintos, dado por la integración del niño a otros grupos de niños fuera del horario de clases en su comunidad, sumado a una mayor presencia de la madre, a diferencia de los menores pertenecientes a los jardines privados, que suelen vivir en condominios con poca integración comunitaria, sumada al hecho de que posiblemente ambos progenitores trabajen. Esto no pasa de ser una simple hipótesis que requiere profundizarse.

$\mathrm{Al}$ analizar la relación existente con la edad, se observa que esta, tal como se esperaba, correlaciona significativamente con cada una de las dimensiones evaluadas, sin 
Tabla 8. Anova medias, dimensiones y quintil de ingreso

\begin{tabular}{|c|c|c|c|c|c|c|c|c|c|c|}
\hline & & $\mathrm{N}$ & Media & d.t. & & $\begin{array}{l}\text { Suma de } \\
\text { cuadrados }\end{array}$ & $\mathrm{gl}$ & $\begin{array}{l}\text { Media } \\
\text { cuadrática }\end{array}$ & $\mathrm{F}$ & Sig. \\
\hline \multirow{5}{*}{ Psicomotricidad } & 162.000 & 59 & 7,1186 & 1,66179 & Inter-grupos & 53,841 & 4 & 13,460 & 3,233 & ,013 \\
\hline & 323.000 & 70 & 7,4143 & 2,32499 & Intra-grupos & 990,825 & 238 & 4,163 & & \\
\hline & 462.000 & 61 & 6,7213 & 2,26665 & & & & & & \\
\hline & 653.000 & 40 & 6,8500 & 1,88856 & & & & & & \\
\hline & 1.285 .000 & 13 & 8,7692 & ,92681 & & & & & & \\
\hline \multirow[t]{5}{*}{ Lenguaje Articulatorio } & 162.000 & 59 & 8,2034 & 4,00336 & Inter-grupos & 54,394 & 4 & 13,598 &, 752 &, 558 \\
\hline & 323.000 & 70 & 9,0857 & 4,48425 & Intra-grupos & 4306,404 & 238 & 18,094 & & \\
\hline & 462.000 & 61 & 9,0820 & 4,21622 & Total & 4360,798 & 242 & & & \\
\hline & 653.000 & 40 & 9,5000 & 4,36184 & & & & & & \\
\hline & 1.285 .000 & 13 & 9,6923 & 3,88125 & & & & & & \\
\hline \multirow[t]{5}{*}{ Lenguaje Expresivo } & 162.000 & 59 & 2,0847 & 1,31684 & Inter-grupos & 13,659 & 4 & 3,415 & 2,055 & 087 \\
\hline & 323.000 & 70 & 2,3143 & 1,25736 & Intra-grupos & 395,526 & 238 & 1,662 & & \\
\hline & 462.000 & 61 & 2,1148 & 1,33039 & Total & 409,185 & 242 & & & \\
\hline & 653.000 & 40 & 2,2750 & 1,26060 & & & & & & \\
\hline & 1.285 .000 & 13 & 3,1538 & 1,21423 & & & & & & \\
\hline \multirow[t]{5}{*}{ Lenguaje Comprensivo } & 162.000 & 59 & 3,2881 & 2,44985 & Inter-grupos & 46,441 & 4 & 11,610 & 2,008 & ,094 \\
\hline & 323.000 & 70 & 4,1571 & 2,22395 & Intra-grupos & 1376,432 & 238 & 5,783 & & \\
\hline & 462.000 & 61 & 3,8361 & 2,44391 & Total & 1422,872 & 242 & & & \\
\hline & 653.000 & 40 & 3,4250 & 2,61051 & & & & & & \\
\hline & 1.285 .000 & 13 & 4,9231 & 2,28989 & & & & & & \\
\hline \multirow[t]{5}{*}{ Estructuración Espacial } & 162.000 & 59 & 6,9492 & 2,41706 & Inter-grupos & 128,106 & 4 & 32,026 & 4,231 & ,003 \\
\hline & 323.000 & 70 & 7,5286 & 2,98169 & Intra-grupos & 1801,507 & 238 & 7,569 & & \\
\hline & 462.000 & 61 & 8,0328 & 2,65811 & Total & 1929,613 & 242 & & & \\
\hline & 653.000 & 40 & 7,9750 & 2,81468 & & & & & & \\
\hline & 1.285 .000 & 13 & 10,2308 & 3,11325 & & & & & & \\
\hline \multirow[t]{5}{*}{ Viso percepción } & 162.000 & 59 & 5,1695 & 3,65849 & Inter-grupos & 184,496 & 4 & 46,124 & 2,947 &, 021 \\
\hline & 323.000 & 70 & 6,6429 & 4,06125 & Intra-grupos & 3725,019 & 238 & 15,651 & & \\
\hline & 462.000 & 61 & 5,9180 & 3,97196 & Total & 3909,514 & 242 & & & \\
\hline & 653.000 & 40 & 4,9750 & 4,12924 & & & & & & \\
\hline & 1.285 .000 & 13 & 8,3846 & 4,07305 & & & & & & \\
\hline \multirow[t]{5}{*}{ Memoria icónica } & 162.000 & 59 & 6,1186 & 2,41464 & Inter-grupos & 51,738 & 4 & 12,935 & 2,265 & ,063 \\
\hline & 323.000 & 70 & 6,3286 & 2,51789 & Intra-grupos & 1359,159 & 238 & 5,711 & & \\
\hline & 462.000 & 61 & 5,4098 & 2,49250 & Total & 1410,897 & 242 & & & \\
\hline & 653.000 & 40 & 5,6500 & 2,06993 & & & & & & \\
\hline & 1.285 .000 & 13 & 7,1538 & 1,90815 & & & & & & \\
\hline \multirow[t]{5}{*}{ Ritmo } & 162.000 & 59 & 1,8644 & 1,59155 & Inter-grupos & 27,630 & 4 & 6,908 & 3,225 &, 013 \\
\hline & 323.000 & 70 & 2,3286 & 1,51056 & Intra-grupos & 509,777 & 238 & 2,142 & & \\
\hline & 462.000 & 61 & 1,4754 & 1,25972 & Total & 537,407 & 242 & & & \\
\hline & 653.000 & 40 & 1,9750 & 1,44093 & & & & & & \\
\hline & 1.285 .000 & 13 & 2,4615 & 1,56074 & & & & & & \\
\hline \multirow[t]{5}{*}{ Fluidez Verbal } & 162.000 & 59 & 42,9492 & 69,51145 & Inter-grupos & 60098,318 & 4 & 15024,579 & 3,142 &, 015 \\
\hline & 323.000 & 70 & 65,9857 & 75,09462 & Intra-grupos & 1137971,938 & 238 & 4781,395 & & \\
\hline & 462.000 & 61 & 27,1148 & 42,72162 & Total & 1198070,255 & 242 & & & \\
\hline & 653.000 & 40 & 51,6000 & 77,30652 & & & & & & \\
\hline & 1.285 .000 & 13 & 74,2308 & 102,48427 & & & & & & \\
\hline
\end{tabular}




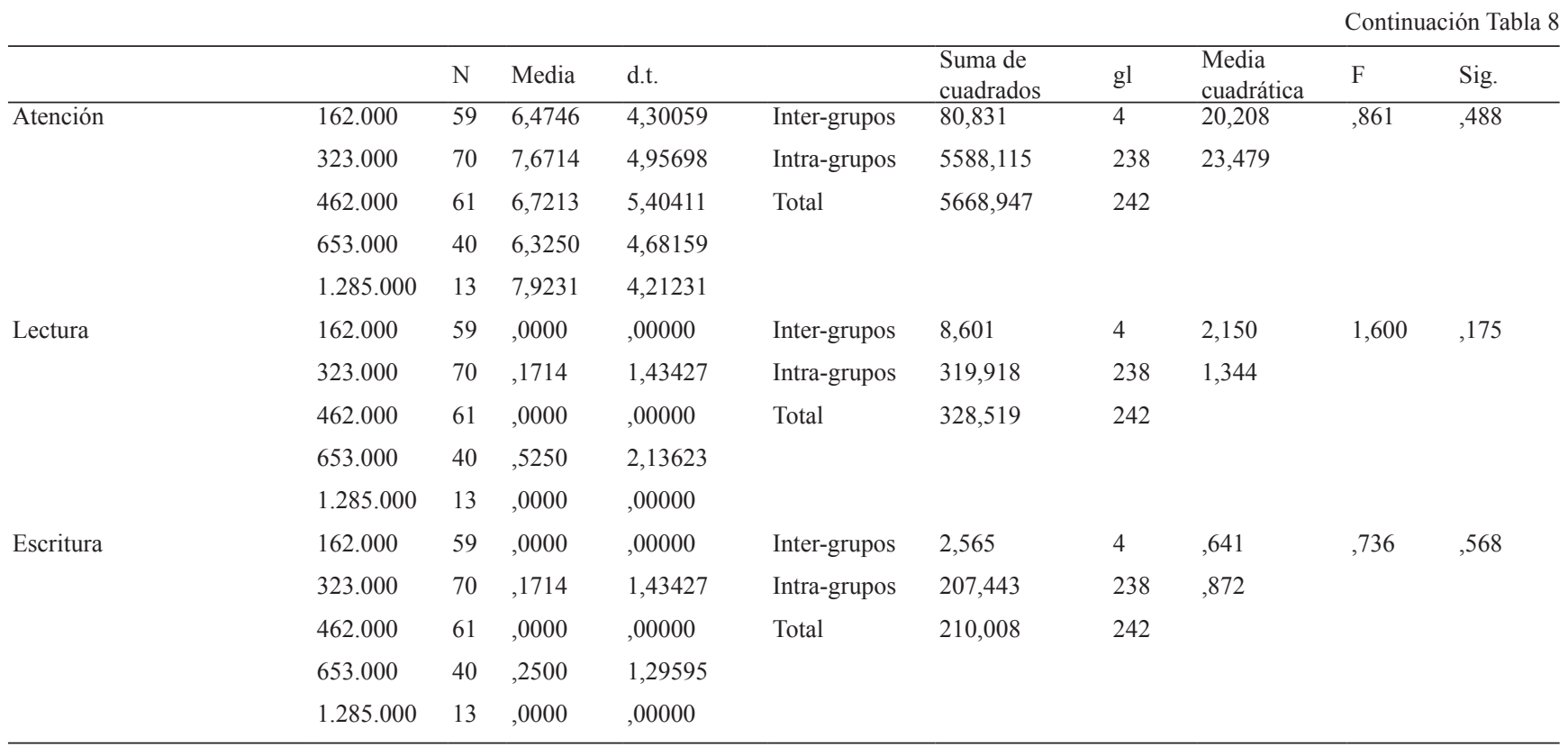

Ptj.
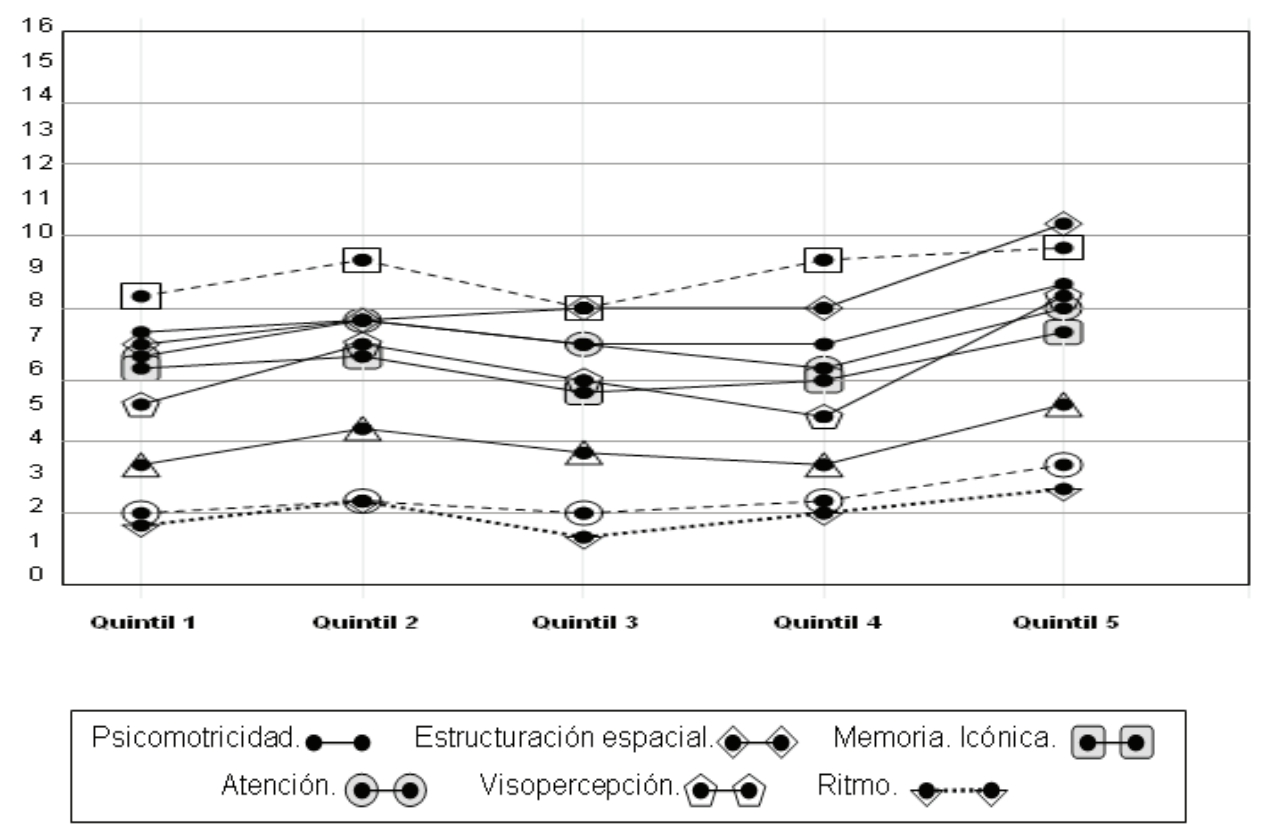

Escalas No verbales

Leng. Articulatorio $\bullet-\bullet$ Leng. Expresivo-- Leng. Comprensivo

Escalas verbales

Figura 2: Medias según dimensión y quintil de ingreso

embargo, el tamaño de la muestra imposibilita el análisis por rango de edad.

A pesar de que el formato de respuesta del instrumento no ha posibilitado el realizar un análisis de su estructura factorial, se encuentra que el instrumento es capaz de discriminar entre grupos de acuerdo a lo esperado teóricamente, es decir, por grupos de edad, dado que la varianza de las medias entre niveles de rango etáreo son estadísticamente significativas.

Tal como se hipotetizaba, las medias en las distintas dimensiones se incrementan a medida que la edad también lo hace. El comportamiento de los ítems es el esperado desde el punto de vista de una escala de desarrollo, ya que se van incrementando las medias de acuerdo con el 
rango etáreo. Resultados similares fueron obtenidos por Portellano, Mateos \& Martínez (2000) y en la validación del instrumento en Perú (Guerrero, 2006).

Los resultados muestran que las escalas más difíciles (con medias más bajas en relación a la cantidad de ítems por dimensión) corresponden a las que evalúan la función rítmica y las que evalúan el nivel de desarrollo de la lectoescritura. Tanto la muestra antofagastina como la de Lima y la española obtuvieron bajos puntajes en la escala de ritmo.

Algunos autores han planteado que las zonas de integración parieto-temporo-occipitales que permiten la diferenciación de las letras similares -esenciales para el proceso de lectura y escritura- se desarrollan a cabalidad después la edad de 6 años (Luria, 1986; Solovieva y Quintanar, 2000), rango etáreo que no fue abarcado en la muestra utilizada en la validación del instrumento. Esto explicaría los resultados obtenidos por la muestra en la dimensión lectura y escritura.

Independiente de las diferencias estadísticamente significativas en las dimensiones Psicomotricidad, Estructuración Espacial, Viso percepción, Ritmo y Fluidez Verbal, respecto de los Quintiles de Ingreso Económico, en términos generales se observa una superioridad general de las medias en todas las dimensiones de los Quintiles de mayor ingreso económico, sobre los de menor ingreso económico. Estos hallazgos guardan congruencia con una serie de estudios (Baydar, Brooks-Gunn, \& Furstenberg, 1993; BrooksGunn, Guo, \& Furstenberg, 1993; Noble, Norman y Farah, 2005) que señalan que la variable estatus socioeconómico ejerce una fuerte influencia en funciones cognitivas como memoria y habilidad verbal funcional, logros destacados en escalas de tareas y en el desarrollo general de habilidades cognitivas que permiten el logro de tareas durante la niñez y los años posteriores respectivamente.

Limitaciones de este estudio pueden encontrarse en su transversalidad, ya que hubiese sido interesante la realización de un estudio longitudinal, bajo un diseño de medidas repetidas, que permitiese evaluar la madurez en distintos periodos de tiempo, además de no poder haber realizado una segunda evaluación a similar muestra a fin de evaluar su confiabilidad.

Se ha comprobado la validez del constructo Madurez Neuropsicológica así como la unidimensionalidad de la escala a través de la revisión por criterio de jueces expertos; por la comprobación de la mayor parte de las hipótesis expuestas al inicio de la investigación y por la alta correlación existente entre las sub-escalas del test. Sin embargo la carencia de instrumentos similares en Chile no ha permitido evaluar la validez convergente del instrumento.

Finalmente, los hallazgos anteriormente expuestos permiten concluir que el Cuestionario de Madurez Neuropsicológica Infantil es un instrumento válido y confiable para la evaluación del constructo unidimensional de desarrollo neuropsicológico, siendo este capaz de discriminar entre rangos de edad, obteniéndose rendimientos más altos a medida que la edad avanza, debido a la consolidación de las funciones superiores del sistema nervioso y su sustento neurológico.

\section{Referencias}

Akhutina, T., Vygotsky, L.S, y Luria, A.R. (2002). La formación de la neuropsicología. Revista española de Neuropsicología, 4, 108-129.

Baydar, N., Brooks-Gunn, J., \& Furstenberg, F. (1993). Early warning signs of functional illiteracy: predictors in childhood and adolescence. Child Development, 64, 815-829.

Bergado, J. \& Almaguer, W. (2000). Mecanismos celulares de la neuroplasticidad. Revista Neurológica, 31, 1074-95.

Brooks-Gunn, J., Guo, G., \& Furstenberg, F. (1993). Who drops out of and who continues beyond high school? Journal of Research on Adolescence, 3, 271-294.

D'Amato, R. C., Rothlisberg, B. A., \& Work, P. H. L. (1998). Neuropsychological assessment for intervention. En Reynolds, C.R., \& Gutkin, T.B. (edts). The handbook of school psychology. (pp. 452-475). New York: Wiley.

Dietrich K., Eskenazi B, Schantz S, Yolton K, Rauh V., Johnson C., Alkon A, Canfield R., Pessah I., Berman R. (2005). Principles and Practices of neurodevelopmental assessment in children: lessons learned from the Centers for Children's Environmental Health and Disease Prevention Research. Environ Health Perspect. 113(10), 1437 - 1446

Ginarte, Y. (2007). La neuroplasticidad como base biológica de la rehabilitación cognitiva. GEROINFO RNPS 2110. 2(1), 1-15. Disponible en la página de la Red Cubana de Gerontologìa y Geriatría http://www.sld.cu/ galerias/pdf/sitios/gericuba/pub._bases_biol._de_reh._cog.pdf

Guerrero, K. (2006). Adaptación del cuestionario de madurez neuropsicológica infantil - cumanin - en una población urbana de Lima. Revista Electrónica del Instituto Psicología y Desarrollo, 3, 1-14.

Huttenlocher, P. \& Dabholkar, A. (1997). Regional differences in synaptogenesis in human cerebral cortex. Journal of Comparative Neurology, 387, 167-178.

Kolb, B. \& Whishaw, I. (2006). Neuropsicología Humana. España: Editorial Médica Panamericana S. A.

Korkman, M. (2001). Introduction to the special issue on normal neuropsychological development in the school-age years. Developmental Neuropsychology, 20, 325-330

Luria, A. (1984). El cerebro en acción. Cuba: Editorial Pueblo y Educación.

Luria, A. (1986). Las funciones corticales superiores del hombre. México: Fontamara.

Maggiolo, M, \& de Barbieri, Z. (1999). Programa de estimulación temprana del lenguaje. Revista chilena de fonoaudiología, 1, 31-40.

Martínez, M. (1999). El enfoque sociocultural en el estudio del desarrollo y la educación. Revista electrónica de investigación educativa, 1(1), Recuperado en abril 29, 2007 disponible en http://redie.uabc.mx/contenido/vol1no1/contenido-mtzrod.pdf.

Noble, K., Norman, F. \& Farah M. (2005). Neurocognitive correlates of socioeconomic status in kindergarten children. Developmental Science, $8,74-8$.

Paterno, R., \& Eusebio, C. (2002). Neuropsicología infantil: sus aportes al campo de la educación especial. Recuperado en mayo 16, 2007 disponible en web Fundación Neuropsicologìa clínica: http://www.fnc. org.ar/pdfs/paterno_eusebio.pdf

Portellano, J., Mateos, R., \& Martínez, R. (2000). Cuestionario de Madurez Neuropsicológica Infantil (CUMANIN). Madrid: TEA Ediciones.

Quintanar, L. \& Solovieva, Y. (2005). Análisis neuropsicológico de los problemas en el aprendizaje escolar. Revista internacional del Magisterio, 15, 26-36.

Rosselli, M., Ardila, A., Bateman, J. R., \& Gúzman, M. (2001). Neuropsychological Test Scores, Academic Performance, and Developmental 
Disorders in Spanish-Speaking Children. Developmental Neuropsychology, 20, 355-373.

Serbin, L. \& Karp, J. (2004). The intergenerational transfer of psychosocial risk: mediators of vulnerability and resilience. Annual Review Psychology, 55, 333-63.

Silver, C., et al., (2006). The importance of neuropsychological assessment for the evaluation of childhood learning disorders NAN Policy and Planning Committee. Archives of Clinical Neuropsychology, 21, 741-744.

Solovieva, Y. \& Quintanar, L. (2000). Método invariante para la enseñanza de la lectura, una aproximación histórico cultural. México: Universidad Autónoma de Puebla.
Solovieva, Y., Quintanar, L. \& Lázaro, E. (2002). Evaluación neuropsicológica de escolares rurales y urbanos desde la aproximación de Luria. Revista española de neuropsicología, 4, 217 - 235.

Thorell, L. \& Wahlstedt, C. (2006). Executive functioning deficits in relation to symptoms of adhd and/or odd in preschool children. Infant and Child Development. 15, 503-518.

Vygotsky, L. S. (1981). The genesis of higher mental functions. En Wertsch J. V., (Ed.). The concept of activity in soviet psychology. Armonk: Sharpe. 